\section{Evidence-based Emergency Medicine}

Edited by Brian H. Rowe, Eddy S. Lang, Michael Brown, Debra Houry, David H. Newman, Peter C. Wyer. 2009. Published by Wiley-Blackwell. Clothbound, 658 pp. US\$275.00.

T his emergency medicine textbook takes a novel approach, in that it does not attempt to discuss all areas of emergency medicine. The areas not covered probably exceed those that are; there does not appear to have been any definitive process to determine why key topics would be included or why they would be absent (e.g., pain, substance abuse, environmental issues, prehospital care, abdominal trauma). Hopefully, future editions will be expanded to cover more clinical conditions.

But enough of what is not there, let's see what is there. To my knowledge, this is the first emergency medicine textbook to show clinicians how to incorporate current pertinent evidence into their day-to-day practice. It does so using a structure that asks relevant clinical questions, then answers those questions by providing the evidence available. Should clopidogrel be used for acute coronary syndrome? Should dexamethasone be given to migraine sufferers before discharge? Such questions are pertinent and relevant to the clinician.

This approach is perhaps the only way to ensure knowledge transfer to clinicians in a timely fashion. Past methods have resulted in a system whereby it has taken an average of 12 years for new evidence to be incorporated into general practice. By providing new information, as well as the way the evidence was obtained, clinicians are more likely to accept knowledge into practice.
Does this textbook tell you how to practise? The answer is no, for evidence-based medicine is a combination of evidence, physician experience and patient expectation. For a clinician seeking "the answer," there may be some frustration. Often, the evidence-based answer to a specific clinical question is that there is no valid evidence one way or another. For most, however, the evidence presented will provide solid support for clinical decision-making. What may surprise many is how often the available evidence disagrees with current practice. This is testimony, once again, to the snail's pace of knowledge transfer that exists.

It is precisely because there is such discordance between practice and available evidence that physicians should read this book from cover to cover. Many may choose to read a chapter after seeing a specific clinical scenario, as this method may optimally anchor new information.

I strongly encourage readers to read the first few (nonclinical) chapters. They will facilitate reading and understanding the clinical material. The nontraditional set-up of the chapters may take a bit of getting used to, but for every one of us it is worth the effort. This textbook provides excellent answers to daily questions. The editors are to be congratulated for their rigour. It should be on everyone's reading list this year.

\section{James Ducharme, MD, CM, FRCP}

Editor-in-chief, CJEM

\section{Competing interests: None declared.}

Keywords: emergency medicine, evidence-based medicine, continuing professional development 